\title{
El trabajo en equipo para promover la participación de los actores sociales
}

\section{The working in team to promote the participation of the social actors}

\author{
Yuraima Matos de Rojas*, María Martina Mejia**, Yvo Antonio \\ Barreto $^{* * *}$ y Maryelin Vethencourt ${ }^{* * * *}$
}

\section{RESUMEN}

El estudio tuvo como propósito: Generar acciones de trabajo en equipo para promover la participación activa de los actores sociales. Metodológicamente se abordó desde la Investigación Acción Participativa, considerando las posturas de Grundy (citado en López; 2001), Astorga y Van Der Bijl (1994), así como López (2001), a través de las fases: diagnóstico, planificación, ejecución y evaluación; acompañados de momentos de reflexión. El diagnóstico arrojó como problema de estudio la falta de participación activa y el trabajo en equipo. Problemática analizada por los propios actores de la escuela y comunidad de Agua Clara, Edo Trujillo. Se planificaron diversas actividades, las cuales se fueron desarrollando desde una participación entre iguales, constituyendo el sello de la investigación-acción. Como resultado se obtuvo: el 75\% de la participación de los actores de la escuela y comunidad, así como talleres que generaron reflexión, acción, formación y transformación de los participantes. Concluyendo que se logró la participación activa desde el trabajo en equipo, transformaciones con la comunidad, para la comunidad y por la comunidad a través de la participación activa e involucramiento de los actores socio-educativos, esperando que sigan participando activamente en beneficio de la transformación social que requiere su comunidad y el país.

Palabras clave: participación, equipo, transformación.

\footnotetext{
*Doctora en Ciencias de la Educación, Docente-Investigadora de la UNESR, Núcleo Valera, Edo. Trujillo, Venezuela. Coordinadora de la Línea de investigación "Investigadores en Acción Social" (IAS), Miembro del PEII Nivel B. Correo Electrónico: yuraimatos01@gmail.com

*** Magister en Gerencia Educativa, Estudiante del Doctorado en Educación, UNERMB, Trujillo, Venezuela. Docente de aula. Participante de la Línea de Investigación IAS, UNESR. Correo Electrónico: mariamdecordero@ gmail.com

*** Magister en Gerencia Educativa, Estudiante del Doctorado en Educación, UNERMB, Trujillo, Venezuela. Docente de aula. Participante de la Línea de Investigación IAS, UNESR. Correo Electrónico: yvobarreto.a@ gmail.com

**** Magister en Administración de la Educación Básica en la UNERMB. Estudiante del Doctorado en Educación, UNERMB, Trujillo, Venezuela. Docente de aula. CorreoElectrónico: mayerajedrez@ gmail.com
}

226 


\begin{abstract}
Aim of the study was: generate actions of work as a team to promote the active participation of the social actors. Methodologically addressed from participatory action research, whereas the positions of Grundy (cited in Lopez; 2001), Astorga and Van Der Bijl (1994), as well as López (2001), through the stages: diagnosis, planning, implementation and evaluation; accompanied by moments of reflection. The diagnosis threw as study problem the lack of active participation and work team. Problems analyzed by the actors themselves of the school and community of Agua Clara, Edo Trujillo. Activities, which were developed from peer involvement, constituting the seal of action research were planned. As result was obtained: $75 \%$ of the participation of the actors of the school and community, as well as workshops which generated reflection, action, formation and transformation of the participants.Concluding that it was the active participation from teamwork, transformation with the community, for the community and by the community through the active participation and involvement of the socio-educational players, hoping to continue participating actively in favors of social transformation which requires the community and the country.
\end{abstract}

Key words: participation, team, transformation.

\title{
Introducción
}

En los últimos años, se ha venido observando gran preocupación por parte del Ministerio del Poder Popular para la Educación, en cuanto al mejoramiento de la calidad educativa y a la participación de los distintos actores responsables del proceso educativo de los estudiantes. Inquietud que ha dado paso a grandes cambios, desde el proceso de gestión hasta la planificación en colectivo con los actores comunitarios (llámese escuela y comunidad). Convirtiéndose en una ventaja que vislumbra la adaptabilidad de la institución a los nuevos entornos, para adelantarse a la evolución de los acontecimientos.

Desde esta perspectiva, se hace necesario favorecer la participación activa de los actores que hacen vida en la escuela y comunidad, donde se trabaje en equipo para elevar la calidad educativa y resolver en conjunto los problemas que le afectan. En tal sentido, el trabajo en equipo lleva a fortalecer la participación, siendo ésta un derecho y un deber establecido en la Constitución de la República Bolivariana de Venezuela a 
través de la Asamblea Nacional Constituyente (1999), Art. 132 donde refiere participar solidariamente en la vida comunitaria; así mismo en la Ley Orgánica de la Educación, según la Asamblea Nacional (2009), se establece la participación social, especialmente en el proceso educativo de los hijos. Participación que de acuerdo con Manzanilla (2005), no es sólo un mecanismo de funcionamiento para la gestión de la organización; es también un proceso de aprendizaje, un medio de formación para los niños/niñas, los maestros/maestras y las familias.

Ante la relevancia del trabajo en equipo para promover la participación activa, se exhorta a todos los involucrados propiciar cambios y transformaciones que den respuesta a los problemas como a las exigencias del entorno y, lograr como indica Montero (2006), las transformaciones con la comunidad, desde la comunidad, para la comunidad y por la comunidad. De allí la necesidad de realizar esta investigación, donde los actores de la comunidad de Agua Clara se involucren activamente para actuar en beneficio de su escuela y comunidad; de tal manera, que fortalezcan debilidades humanas sostenida en la dialogicidad, en la cooperación y solidaridad, en las relaciones afectivas y cognitivas que conduzcan el pensamiento reflexivo, creador y crítico, sobre todo con conciencia de sí mismo y de los otros.

En tal sentido, el propósito general de esta investigación fue "Generar acciones de trabajo en equipo para promover la participación activa de los actores sociales”. Propósito que surgió desde un diagnóstico participativo, que según El Troudi, Harnecker y Bonilla-Molina (2005), propicia la participación de la comunidad para la edificación en colectivo de un conocimiento sobre su realidad, reconociendo los problemas que afectan, los recursos con que cuentan y las alternativas que se pueden aprovechar en beneficio de los participantes.

Realidad que fue develada por los propios actores, según voces de docentes y directivos de la Escuela de Agua Clara, señalando la poca participación y la falta de trabajo en equipo por parte de la comunidad en la realización de las actividades que la escuela planifica. De igual manera, los actores de la comunidad indicaron la poca motivación que existía para dicha participación. Situación que justifica el estudio, 
teniendo presente el propósito de estudio enunciado anteriormente, donde a través de las acciones que se realicen los lleve a trabajar en equipo, involucrarse y participar activamente hacia la transformación social, en el que los sujetos sean capaces de realizar autoaprendizajes, y autogestión en la realización de proyectos que le beneficie.

En este sentido el estudio se abordó desde la perspectiva teóricometodológica del Paradigma Crítico-Reflexivo, considerando a Grundy (citado en López; 2001),el cual concibe la realidad dinámica y cambiante, constantemente transformada por las actividades del hombre; orientado por el método de la Investigación-Acción-Participativa, en sus fases de diagnóstico, planificación, ejecución y evaluación. Pues se espera que los coinvestigadores a partir de las acciones planificadas en colectivo trabajen en equipo y participen activamente en la medida que asumen el compromiso por su autoformación, participación, trabajo en equipo y la autogestión, como herramientas de poder para decidir por sí mismo las decisiones que le afectan y las soluciones a sus propias necesidades.

\section{Perspectiva teórica de la investigación}

Considerando la problemática de estudio se realizó la revisión teórica de los aspectos concernientes con la participación activa, la autoformación y la autogestión, planteamientos teóricos que propiciarán el acercamiento con las actividades comunitarias en la comunidad de Agua Clara.

\section{El trabajo en equipo}

Para definir trabajo en equipo, Matos, Pasek y Rojas (2008: 5), señalan que es necesario aclarar el término de equipo y grupo, por cuanto no son lo mismo, a pesar que son usados como sinónimos. Por lo que las autoras hacen una diferencia entre grupo y equipo: el primero está conformado por las personas que se reúnen o agrupan por una determinada circunstancias, para compartir información o perspectivas. 
Mientras que un equipo, tiene como base el hecho que las personas forman parte de un sólo grupo y poseen un objetivo o meta común, existe interdependencia entre ellas para la realización de sus tareas y actividades, se rigen por un marco normativo preestablecido y, lo más importante es que, cada uno de sus miembros aporta sus conocimientos y habilidades en la consecución de los objetivos propuestos.

Se trata de trabajar mancomunadamente en el logro de los objetivos, y metas propuestas por el colectivo institucional; por lo tanto, se espera conformar un grupo, donde trabajen en equipo para el logro de las transformaciones que requieren cada uno de los actores y la propia institución. Es decir, articular las actividades en conjunto con los actores de la institución y comunidad, en torno a un conjunto de fines, de metas y de resultados a alcanzar. Donde se refleje la interdependencia activa entre los integrantes del equipo, que asuman responsablemente cada función designada para la conquista de lo planificado y así lograr un fin común.

El trabajo en equipo se caracteriza por la comunicación fluida entre las personas, basada en relaciones de confianza y de apoyo mutuo. Se centra en las metas trazadas en un clima de confianza y de apoyo recíproco entre sus integrantes, donde prevalezca la sinergia. Es decir, aunar esfuerzos y disponer las competencias de cada cual en torno a un objetivo común, generando un todo que es mayor que la suma de sus partes.

Cabe referir a Shermerhom (2008:196), para quien el trabajo en equipo significa "trabajar de manera participativa con el fin de lograr un propósito frente al cual todos sus miembros son responsables". Sabiendo que cada persona posee habilidades, destrezas, conocimientos, saberes que se pueden complementar y, así contribuir al mejoramiento de la productividad y de la calidad de la vida de los actores participantes. Pues se trata que los participantes intercambien sus experiencias, respetando sus roles o funciones para conseguir objetivos comunes al participar activamente en el logro de las actividades planificadas. 


\section{La Participación Activa}

Para señalar lo qué es la participación activa, se requiere en un primer momento, conocer la etimología de la palabra participación, que proviene del latín “partemcapere", significa "tomar parte". Diversas son las concepciones que se le dan a la palabra participación, desde lo señalado en los diccionarios, en los textos, como en los estamentos legales de la nación. Ahora bien, de acuerdo con el Diccionario de la Manual de la Lengua Española (2009) consiste en "la acción y efecto de tomar parte de algo"; también recibir parte de algo, compartir, tener las mismas opiniones, ideas. Implica, entonces, la capacidad de acceso de los miembros de una organización, de un colectivo, a la toma de decisiones que afecta a esa organización.

En lo que respecta a la Constitución Bolivariana de Venezuela, de acuerdo con la Asamblea Nacional Constituyente (1999), el término participación está implícito como un derecho social y dinámico que tienen los ciudadanos de forma consciente en los procesos decisorios de las organizaciones que afectan sus vidas. En ese sentido, desde las revisiones que se han realizado al término y, desde su finalidad, se generan diversas definiciones que merecen ser citadas. Para Sánchez (2001), participar, es tomar parte o compartir, y si es necesario, tomar la parte que a la persona le corresponde. Y esto, no sólo al nivel de actitud sino de hechos con conductas concretas en la entidad que corresponda. Esto demuestra en determinadas circunstancias, que la participación es un requisito para el desarrollo de una organización.

Para Matos, Pasek y Rojas (2014), la participación es vista como un verdadero acto de intervenir directa y activamente en las acciones, asumirla como una responsabilidad compartida, tanto en lo que se refiere al esfuerzo como al disfrute de los beneficios. Por lo que la participación consiste en la intervención de las partes interesadas en la realización y en el disfrute de los resultados, pues sin ésta, no puede haber armonía en la búsqueda de soluciones a hechos concretos. 
Como se viene apreciando, la participación es un derecho y un deber, especialmente en el proceso educativo los niños, niñas y jóvenes. La participación puede ser provocada y organizada, sin que esto signifique necesariamente manipulación, pues es una forma de inducir a las personas para que se involucren en las actividades planificadas y ejecutadas por la escuela. De allí, la necesidad de promover la participación en los diferentes espacios a través del trabajo en equipo, en donde todos se involucren y apropien de las funciones y actividades a cumplir

Desde esta perspectiva, se puede decir que la participación activa es un verdadero acto de intervenir directa y activamente en las acciones, es asumirla como una responsabilidad compartida, tanto en lo que se refiere al esfuerzo como al disfrute de los beneficios. Más aún, como señala D’Angelo (2005), la capacidad y la actividad de las grandes mayorías para actuar en la toma de decisiones, en las relaciones de poder y de influencia en distintos niveles del desarrollo social.

Por lo tanto, al propiciar actividades que lleve a los actores a trabajar en equipo se estaría garantizando, de alguna manera, la participación activay corresponsable entre los actores sociales que hacen vida en la escuela y comunidad, para que se empoderen y hagan posible procesos de autotransformación y autogestión en el desarrollo de su comunidad. Maldonado (2005), señala que se trata de lograr una escuela transformadora, liberadora, productora de transformaciones en las cuales participen los actores sociales, siendo indispensable introducir cambios en la práctica pedagógica, en la organización escolar y en las formas de participación con la comunidad.

Una participación activa y consciente de los actores sociales de la comunidad, que como señala Freire (1993), puedan transformar la realidad y transformarse a sí mismos desde un proceso continuo que implica la praxis, es decir una relación dialéctica entre acción y reflexión, que implica según este autor una inserción crítica e la historia. 
Yuraima Matos de Rojas, María Mejia, Yvo Barreto y MaryelinVethencourt

Telos Vol. 20, No. 2 (2018). 226-247

\section{Recorrido metodológico}

El recorrido o ruta metodológica realizada para efecto del estudio, estuvo orientada por el Paradigma crítico-reflexivo, el cual según Grundy (citado en López; 2001), tiene un interés fundamental por la emancipación y la potenciación para comprometerse en una acción autónoma que surge de intuiciones auténticas, críticas, de la construcción social de la sociedad humana. La ciencia se genera de la interacción, la participación y el diálogo intersubjetivo que propician la reflexión sobre las contradicciones sociales y la consecuente acción transformadora. El saber que se construye puede estar en distintos niveles, uno de los cuales es la generación de teorías críticas, las cuales se refieren a las personas y la sociedad y tratan de explicar cómo actúan la restricción y la deformación para inhibir la libertad.

Desde este paradigma, se aborda el método de la Investigación Acción Participativa, que según Corey (1953, Citado en Pérez, 1994), la define como "El proceso por el cual los prácticos intentan estudiar sus problemas científicamente con el fin de guiar, corregir y evaluar sistemáticamente sus decisiones y sus acciones". Por su parte Kemmis y McTaggart (1988), señala que es una forma de indagación autorreflexiva, emprendida por participantes en situaciones sociales con la finalidad de mejorar su práctica social o educativa, así como la comprensión de las mismas.

En la investigación acción-participativa, se da un proceso o conjunto de acciones que deben realizarse colectivamente por el conjunto de personas, grupo o comunidad que intenta analizar y transformar su realidad social, buscando los medios apropiados para su transformación. Transformación que se logra desde la coparticipación de los sujetos involucrados, como lo plantea D’Angelo (2005), una coparticipación estructural de los actores involucrados desde el inicio mismo de la gestación del proceso de transformación y, de modo determinante en cada una de sus etapas. Coparticipación que se llevó a efecto durante el periodo del 2015 cuando se comenzó el estudio, hasta finales del año 2016, cuando los investigadores se retiraron del contexto de estudio para hacer su respectivo análisis de los acontecimientos. 
Para efecto del estudio se abordaron las fases que plantean Astorga y Van Der Bijl (1994), así como López (2001), del siguiente modo: (a) diagnóstico, (b) planificación, (c) ejecución y (d) evaluación.

1era. Etapa de Diagnóstico:En la cual se propició la participación de los actores de la escuela y comunidad, pues como señalan Martí, Pascual y Rebollo (2005), así como Latorre (2007), mediante la participación los agentes sociales implicados y la aplicación de técnicas adecuadas permite construir entre todos un diagnóstico de la realidad de la comunidad, en este caso de la Escuela de Agua Clara. La base de este diagnóstico la constituyeron las aportaciones, las reflexiones y la visión de una parte importante de la propia comunidad que convive con la situación problema y que es la primera interesada en su resolución. Etapa que llevó a efecto las siguientes acciones:

- Selección de la comunidad.

- Acercamiento y solicitud de permiso para realizar la investigación.

- Descripción del contexto donde se realizó estudio (escuela, comunidad).

- Identificación y clarificación de la situación o problema que se quiere investigar.

- Análisis del problema

2da. Etapa de Planificación: La planificación según Blández (2000), tiene como objetivo elaborar un plan de acción, que responda al enfoque teórico de la investigación. Es el momento en el que se toman un conjunto de decisiones sobre las estrategias que se van a utilizar. Pues, como señala Carr (2002), cuando se planifica, lo que se está haciendo es proyectar un plan para el futuro teniendo en cuenta las experiencias.

En esta etapa de planificación se inició el segundo ciclo del proceso de la investigación acción, donde investigadores, coinvestigadores y equipo de facilitadores aportaron sus ideas para la elaboración del plan general, del cual se desglosaron los planes de acción y los reajustes en la medida que se fueron ejecutando. El plan fue elaborado en consenso por los actores de la escuela, la comunidad y los 
investigadores, teniendo en cuenta la flexibilidad del mismo según las necesidades e intereses de los propios actores comunitarios.

3era. Etapa de Ejecución:También llamada de acción, donde se ejecutó lo planificado, fue un momento crucial de la investigación, pues se trataba de accionar para lograr lo planificado con los actores participantes. Etapa que según Véliz (2008), constituye el trabajo de campo, en la cual se desarrollan las técnicas de recolección de datos, como la observación participativa, los diarios de campo y las entrevistas semiestructuradas. De igual manera, en esta etapa se transcribió, organizó, y sistematizó la información. El análisis se ejecutó a partir de las descripciones y, de alguna manera, contrastada con teóricos, que enriquecen el análisis de la misma. Es de resaltar que, durante esta etapa se llevaron a cabo procesos de reflexión en la medida que se fueron ejecutando los planes de acción, originando así cambios en correspondencia con las necesidades de los participantes.

4ta etapa de Evaluación:También denominada de reflexión, es la que Latorre (2007) refiere como, el reflexionar críticamente sobre lo que sucedió, intentando elaborar una cierta teoría situacional y personal de todo el proceso. A la vez que Blández (2000), es un modo de pensar que implica profundizar, analizar, estudiar, meditar sobre algo detenidamente, con atención, con cuidado. En este apartado se valoraron las acciones ejecutadas tomando en cuenta los propósitos específicos del estudio, las acciones ejecutadas y los logros obtenidos.

Considerando el proceso metodológico de la Investigación Acción Participativa, los/las investigadores(as) fueron sistematizando cada una de las actividades desarrolladas, así como las expresiones señaladas por los actores participantes, las cuales fueron sustentadas o confrontadas con los teóricos, realizando para el proceso de análisis, la triangulación entre lo señalado por los participantes, los teóricos y el análisis de los y las investigadores(as), dando así el carácter científico al estudio. 


\section{Acciones transformadoras generadas en la investigación}

Durante la ejecución de cada una de las actividades desarrolladas se trazó como objetivo la formación teórica y práctica, en donde los actores participantes fueron los propios protagonistas de sus aprendizajes, acompañados por los investigadores y un equipo de especialistas para crear y recrear conocimientos desde un enfoque gnoseológico con carácter dialógico. Pues Rodríguez (2003), indica que el carácter gnoseológico de la educación está estrechamente ligado a su carácter dialógico; por lo tanto, las actividades fueron denominadas encuentros, donde los sujetos durante el interactuar sobre temáticas determinadas fueron aportando ideas, promoviendo así la disertación, la problematizaron, la crítica, la reflexión y construcción de sus propios conocimientos, los cuales podrían llevar a la práctica en su accionar diario.

Cabe resaltar que las expresiones se colocan entre paréntesis acompañadas de los códigos de D (Docente), Di (Directora), R (Representante), Junta Comunal (JC), con su respectivo numeral, a manera de resguardar su confidencialidad.

\section{Encuentro "Investigando desde la realidad de los acontecimientos}

El encuentro denominado "Investigando desde la realidad de los acontecimientos", fue acordado por los actores comunitarios de Agua Clara en una reunión previa. Actividad de planificación realizada en consenso con los propios sujetos participantes para tener conocimiento sobre lo que era Investigación Acción. El mismo se estructuró en tres partes, pues se hizo práctico, en el que se establecieron comisiones de trabajo para llevarlos a organizarse en grupo en primera instancia. Cabe señalar que este tipo de investigación según Pérez, Barquín y Angulo (1999), permite que se involucren los participantes en la planificación de la acción (en razón de la reflexión), en la realización de sus planes en su propia acción (praxis).

El propósito de la reunión fue "Propiciar el intercambio de saberes en torno a los conceptos y procedimientos básicos de la Investigación Acción Participativa (IAP), como herramienta para transformar la realidad". De igual manera, se les motivó 
para que expresaran con libertad sus propias ideas e inquietudes, ya que todos somos coinvestigadores y estamos en proceso de compartir y construir nuevos saberes. Se observó que en un principio existía cierto temor en participar; sin embargo, en la medida que se fue dando la reunión, los presentes fueron ganando confianza.

Se dialogó sobre lo que sabían de la Investigación Acción Participativa (IAP), oyéndose algunas expresiones, por parte de la docente D8: "es investigar en la medida que se actúa para resolver problemas", para D45: "es la que se realiza en las comunidades donde todos participan para resolver problemas en colectivo": Expresiones que llevaron a mostrar la lámina donde aparecía la definición de Elliot (2000), la cual decía "Investigación Acción es el estudio de una situación social con el fin de mejorar la calidad de acción dentro de la misma", señalando los aspectos coincidentes entre lo que decían y lo señalado por Elliot.

En la medida que los investigadores iban explicando el material sobre IAP, surgieron preguntas que los llevaron a participar y expresar sus inquietudes, en cuanto a ¿cómo se sienten?, a lo que respondieron al unísono "bien porque estamos aprendiendo cosas nuevas". De igual manera, se fueron trabajando aspectos como las fases de la IAP, sugerencias, aspectos a considerar, técnicas e instrumentos, entre otros, que llevaron a realizar actividades en pequeños grupos que luego compartieron con el resto de los participantes.

Para finalizar se les pidió reflexionar sobre cómo les había parecido la actividad, a lo que se oyeron expresiones de agrado, tales como: D25 "me gustó porque aprendimos algo nuevo", para D1: "bueno me gustó porque yo tenía cierto conocimiento y aclaré algunas dudas", en la voz de la Di: "nos dimos cuenta que la IAP no es tan difícil como nos dicen cuando estudiamos". Mientras que una R45: "me agradó la actividad porque fue dinámica y muy activa, de verdad que aprendimos y compartimos", a lo que la D20: "yo la voy a poner en práctica no sólo en mi salón de clase con mis estudiantes sino también en la comunidad donde vivo, es más fácil actuar en conjunto para poder resolver los problemas que nos aquejan". Para un 
miembro de la JC: "las láminas estaban muy fácil de leer y la dinámica nos permitió participar a todos", para la R28: "los facilitadores lo hicieron muy bien, pues se le nota que tienen dominio en el tema", entre otras expresiones que permitieron conocer sus sentires.

Desde este reflexionar sobre lo realizado en los encuentros, los participantes reflejaron que los conocimientos adquiridos les servirá para ponerlos en práctica en los diferentes espacios, tales como escuela y comunidad. En palabras de Freire (2005), construir conocimientos es alcanzar un estado de comprensión, respecto a temas y situaciones de interés personal que permite al individuo actuar sobre su entorno, sobre sí mismo y su realidad.

Es necesario señalar que la mayoría de los grupos organizados cumplieron con las actividades asignadas, como fueron: logística, material de apoyo, invitación del personal, compartir, entre otras que se fueron dando en la medida de los encuentros. Lo cual les permitió señalar a la R25: "me siento bien, porque mi comisión cumplió el 100\%”, por lo que otra de las participantes, R18 expresó "aunque mi comisión no fue muy participativa, logramos nuestro objetivo al final". Se trata que todos desde su experiencia, aprecien el valor del trabajo en equipo y la participación, como refieren Matos, Pasek y Rojas (2008), se estima la preeminencia de la conformación de grupos sobre la base del trabajo en equipo, en aras de integrar la producción de saberes a los nuevos horizontes de la sociedad que requiere el país.

\section{Encuentro el Trabajo en Equipo como estrategia para lograr la participación}

Encuentro que tuvo como objetivo "Reflexionar desde el accionar cómo el trabajo en equipo promueve la participación y la unión de esfuerzos para el logro de metas u objetivos comunes". Planificación que contó con la participación de invitados especiales, quienes desde sus conocimientos en el área de administración propusieron ideas significativas para el desarrollo del mismo.

Los participantes se organizaron en equipo y comenzaron a dialogar sobre el material y las imágenes presentadas. Pasado el tiempo establecido se procedió a oír cada equipo de trabajo sobre la reflexión realizada. Un equipo señaló: D6: "no 
podemos vivir aislados uno del otro, porque necesitamos del otro para poder avanzar e interactuar y compartir"; otro equipo dijo Di15 "me gustó el encuentro, se trata de que aprendamos a trabajar en equipo y así unirnos más, participar para transformar nuestra realidad institucional".

Expresiones que se relaciona con lo presentado por Hartzler y Henry (1999), al indicar que los equipos necesitan la participación de todos sus miembros para el logro de las metas de la organización. Así como lo señalado por un docente, cuando expresó "tenemos que aprender a trabajar en equipo, pues muchas veces somos tan individualistas que nos encerramos en nuestras aulas y poco compartimos". Desde este reflexionar, se puede decir que los participantes están conscientes de la necesidad de trabajar en equipo, lo que de acuerdo con García, Mena y Sánchez (2011), el reflexionar sobre su propia situación conduce a la toma de conciencia y generar nuevos conocimientos profesionales desde su propio accionar en sí y/o sobre sus condicionantes.

Encuentro que permitió, no sólo reflexionar sobre la necesidad del trabajo en equipo, sino también de participar activamente para el logro de las metas u objetivos. De allí que se realizaron ejercicios para internalizar lo qué es un grupo y el trabajo en equipo, su diferencias y características. Así mismo, se establecieron normas para trabajar en equipo, haciendo énfasis en la comunicación, el liderazgo, la responsabilidad, el respeto por los acuerdos, las metas u objetivos, la planificación, la motivación, como elementos necesarios para el logro de un verdadero trabajo en equipo.

En este sentido, se hace énfasis en la necesidad de internalizar lo aprendido, lo cual para Matos, Pasek y Rojas (2008), internalización e integración que amerita una comunicación abierta, un compartir, un interactuar, un liderazgo efectivo, el apoyo mutuo, la toma de decisiones compartidas, una sinergia en un ambiente cargado de armonía y valores que direccionen el trabajo en equipo para lograr no sólo lo 
planeado sino también una mayor participación activa entre los actores sociales en beneficio propio, de la escuela y comunidad.

Considerando las reflexiones, así como, las experiencias de los participantes, se puede decir que se obtuvo efectividad en el encuentro, en cuanto a que se organizaron varios equipos de trabajo dentro de la misma investigación, quienes desde su accionar realizarían diversos proyectos, quedando de acuerdo que todos deberían ser sistematizados y socializados en las reuniones a convenir. Se apreció gran interés y participación entre los actores participantes, generando ideas y actividades a realizar. Desde la misma se generó el siguiente encuentro para aprender a elaborar proyectos.

\section{Encuentro "Aprendiendo a elaborar Proyectos Socio-comunitarios"}

Este encuentro se planificó en conjunto con los coinvestigadores participes en la investigación, pues los mismos señalaron que hoy día para gestionar cualquier problema comunitario tenían que saber elaborar proyectos de índole sociocomunitarios. El encuentro tuvo como objetivo "Propiciar el intercambio de saberes y experiencias en la elaboración de proyectos socio-comunitarios como una herramienta de trabajo para el logro de un bien común”.

Para efecto del mismo, se organizaron mesas de trabajo que les permitió compartir información y experiencias desde el material asignado. Cada grupo fue aportando ideas sobre el material trabajado, observándose la participación de los agentes sociales, pues como señala Krause (2002; citado por Scribano; 2007: 192), "fomentar la participación activa y responsable de la comunidad;...facilita el acceso a las oportunidades de crecimiento y desarrollo".

En la medida que se fue desarrollando la actividad, se dio la revisión teórica sobre los pasos para elaborar los proyectos socio-comunitarios, originando la participación, la reflexión desde sus haceres y saberes, así como la reconstrucción de nuevos aprendizajes. Para internalizar lo aprendido durante el encuentro se formularon interrogantes, tales como: Qué aprendimos durante el encuentro, a lo que respondió uno de los participantes, D12 "lo social es la comunidad que tiene problemas de 
diversa índole, debemos tomarlos en cuenta para buscar soluciones en colectivo", otra de las D7 indicó "para elaborar los proyectos debemos tener en cuenta una serie de elementos que van a contribuir a su buen diseño", otra expresión fue la enunciada por Di30: "los proyectos sociales deben ser trabajados en conjunto con las comunidades, pues todos somos investigadores y nosotros como docentes podemos orientarlos, se trata de trabajar en equipo". Para Pérez (2002), los proyectos sociales hacen referencia y se orientan a la resolución de problemas para satisfacer las necesidades básicas del individuo.

Desde las expresiones señaladas, la reflexión constituye un proceso social y colectivo, esencial para el desarrollo de los actores sociales, quienes vieron la necesidad de aprender a realizar proyectos desde la unión, el trabajo en equipo y para fortalecer aprendizajes. Como señala Alanís (2004), al referir que desde la reflexión se genera un aprendizaje más sólido y con ella se desprende la confrontación de lo que se sabe con lo que exige el problema de estudio. Por lo tanto, se trata de fomentar la reflexión crítica y cultivarla durante el accionar de todo docente, a la vez, que estos se formen para ponerla en práctica en sus espacios de aprendizaje.

Es necesario señalar que desde actividad realizada, se generaron cuatro (4) proyectos: patrulla escolar, la recreación como espacio de formación, organizando espacios de aprendizaje y la integración de las comunas en las actividades escolares. Proyectos que fueron liderizados por los actores sociales de la escuela, con la participación de representantes y orientados por los investigadores. Valorizando cada actividad como proceso de cambio, tal como lo refleja la directora, al señalar "estamos camino hacia un cambio de cultura más productiva y sustentable en el transcurso de tiempo, ya estamos aprendiendo a trabajar y participar como equipos".

En este cambio se visualizan valores que llevan a generar procesos de transformación, que como señalan Chávez e Ibarra (2016: 149):

Si se contemplan los valores como parte de un cambio se hablaría entonces de una transformación en la cultura de la organización, pero se trata de una meta con carga social (sustentabilidad), por lo que es 
necesario transmitir a los seguidores la visión de una dinámica de desarrollo que englobe tanto a la organización como el entorno.

De allí la necesidad de promover entre los actores sociales, el liderazgo como accionar de quien dirige cada proyecto, quien orienta las actividades a desarrollar con su equipo de trabajo, en un ambiente colaborativo y participativo, abriendo la oportunidad que se intercambien ideas a la vez que se reflexiona para generar cambios hacia el logro de metas grupales, organizacionales e individuales. En este sentido, se trata de dejar huellas de satisfacción, compromiso y apropiación por mejorar y actuar en colectivo hacia una mejor calidad educativa.

\section{Encuentro "la autogestión para trabajar en equipo"}

Encuentro que tuvo como propósito "dialogar sobre lo que implica la práctica autogestionaria en el trabajo en equipo". Ello consistió en hacer una breve recapitulación de todos los encuentros, reflexionando sobre los saberes adquiridos y los cambios que se han logrado desde los mismos. Mora (1991) indica que es necesario un proceso de capacitación, pero no en el sentido de un mero aprendizaje sino en función de los intereses del grupo, la cual debe partir de la explicación objetiva de los fenómenos en forma integrada y dialéctica. De igual manera, indica que debe construirse en forma histórica, con el fin de hacer resaltar las contradicciones como sucesos dinámicos de la sociedad, y que tienda a su vez a la transformación de ella.

Ahora bien hablando de la autogestión comunitaria, los participantes señalaron R 24 "es asumir con autonomía la realidad que tenemos para actuar en beneficio de ella", otra de las expresiones fue la de JC "todos los encuentros que hemos realizados están en función de la autoformación y concienciación de las acciones que podemos realizar en equipo en pro de nuestra comunidad". Cabe indicar a El Troudi, Harnecker y Bonilla-Molina (2005), quienes señalan que este proceso en comunidad se conduce con autonomía en coordinación con los intereses y acciones con otros grupos. 
Para referir las concepciones que tienen los autores sobre la autogestión, se hace necesario comenzar por descifrar la terminología, donde "Auto" es un prefijo que significa "uno mismo", o "por sí mismo" y "Gestión” se define como administrar o también como hacer diligencias para conseguir algo, como puede ser un producto, bien o servicio. Pero autogestionario no significa autosuficiente. La idea de autogestión, según Quiroga, Quevedo y Chiriboga (2003), persigue el poder para decidir por sí mismo sobre las decisiones que le afectan.

En este sentido, autogestión es un proceso mediante el cual se desarrolla la capacidad individual o de un grupo para identificar los intereses o necesidades básicas que lo son propios y que a través de una organización permita defenderlos expresándolos con efectividad en la práctica cotidiana, basándose en una conducción autónoma y en una coordinación con los intereses y acciones de otros grupos, este concepto por supuesto que lleva implícito de planificación, democracia participativa y desarrollo sustentable.

Durante el encuentro se apreció el interés de los participantes por ahondar y aclarar el termino, por lo que se les presentó lo señalado por El Troudi, Harnecker y Bonilla-Molina (2005), quienes señalan que la autogestión implica la participación de un grupo de personas en la dirección del desarrollo de la sociedad en términos globales, que lleve no sólo al intercambio sino también a una transformación de la organización social y de la vida. Proceso que de acuerdo con uno de los participantes “implica la participación activa de sus miembros en la medida que se genera la comunicación, la toma de conciencia, toma de decisiones y adopción de responsabilidades; para desarrollar así las potencialidades de los participantes”.

Expresión que llevó a reflexionar a los demás participantes, al referir Di: “eso es lo que vamos a hacer desde el trabajo en equipo para la realización de los diversos proyectos que estamos realizando", por otra parte una D4 señaló "que bueno es lo que estamos aprendiendo, ahora podemos decir que a través del trabajo en equipo podemos elaborar diversos proyectos que nos lleva a gestionar para lograr las acciones 
planificadas, y así estaremos participando todos", a lo que un R22 dijo "de eso se trata de participar activamente para lograr lo que nos proponemos como equipo, hasta lo podemos hacer en nuestra comunidad y hogar".

Diálogo que llevó a la reflexión y al compromiso en que los miembros de todo grupo u organización deben estar preparados para realizar la autogestión en función de alcanzar sus metas u objetivos. La capacitación para una autogestión efectiva conduce a sus participantes a tomar conciencia de lo que realmente desean. Por lo que se podría señalar la autogestión académica, que según Revueltas (2008), es la toma de conciencia, lo cual debe asumirse como el hecho teórico-práctico para el ejercicio de una conciencia colectiva en acción.

Cabe referir que los actores sociales participantes de la Comunidad de Agua Clara que participaron en las acciones transformadoras, asumen que se han empoderaron del proceso de formación para actuar, cambiar y transformar la realidad que de alguna manera les estaba afectando. Postura que los lleva a reflexionar y tomar conciencia que, en la medida que se formen podrán participar activamente en las actividades de la escuela y comunidad y así, generar el proceso de transformación de la realidad desde su accionar como verdaderos equipos de trabajan que participan activamente para lograr las metas u objetivos trazados.

\section{Reflexiones generales}

La realización de este estudio permitió que se generara la investigaciónacción-formación, triangulo que según Lewin (citado por Latorre; 2007), es el triángulo de la investigación, de la acción y de la formación como elementos esenciales para el desarrollo profesional, con la finalidad de mejorar la praxis a través de ciclos de acción y reflexión. En este sentido, se logró en cierta medida que se organizaran en equipos de trabajo para fomentar la participación activa en cada uno de los encuentros, donde un gran número de actores comunitarios de Agua Clara se comprometieron por continuar formándose y accionando para transformar su propia realidad social. 
Desde ese compromiso, se acordó que, los actores comunitarios (escuelacomunidad) deben valerse de herramientas y métodos de trabajos que los lleve a integrarse y participar activamente en cada una de las actividades que planifiquen en colectivo. Por lo que asumieron el compromiso de autogestionar un espacio que les permita reunirse para opinar y expresar sus expectativas, inquietudes y necesidades; de igual manera, para seguir realizando encuentros formativos y proyectos en beneficio de la comunidad.

En la misma forma, se acordó la incorporación de los padres y representantes en tareas de enseñar, orientar y formar a sus hijos de manera sistemática, constituyendo un reto para la nueva escuela, donde éstos tengan un papel relevante junto a los docentes con respecto a las actividades que se programen en la institución, esperando así lograr cambios actitudinales que les permitan desarrollar conductas en pro del proceso educativo y de los participantes en general.

La interpretación crítica de las experiencias, llevó a la construcción y reconstrucción de acciones, favoreciendo el trabajo en equipo, así como la producción de saberes, conocimientos y aprendizajes significativos desde la apropiación crítica de lo vivido y, comprendido teóricamente desde una perspectiva transformadora.

En este sentido, se espera que desde ésta experiencia, los actores de la comunidad de Agua Clara, sean capaces seguir generando acciones como equipo de trabajo y con ello la participación activa del colectivo. A la vez que aprovechen al máximo los conocimientos adquiridos, y de esta manera, participen activamente en beneficio de la transformación social que requiere su comunidad y el país.

\section{Referencias Bibliográficas}

Alanís, Antonio (2004). Formación de Formadores. Fundamentos para el desarrollo de la investigación y la docencia. Editorial Trillas. México.

Asamblea Nacional Constituyente (1999). Constitución Nacional de la República Bolivariana de Venezuela. Gaceta Oficial 36.860. Venezuela.

Asamblea Nacional (2009). Ley Orgánica de Educación. Gaceta Oficial 5.929 Extraordinario. Venezuela. 
Astorga Alfredo y Van Der Bijl, Bart. (1994). Manual del Diagnóstico Participativo. Editorial Humanitas-Cedepo. Argentina.

Blández, Julia (2000). La Investigación-acción. Un reto para el profesorado. Publicaciones INDE. España.

Carr, Wilfred (2002). Una Teoría para la Educación. Hacia una Investigación educativa crítica. (Tercera edición). Editorial Morata. España.

Chávez, José e Ibarra Juan (2016). Liderazgo y cambio cultural en la organización para la sustentabilidad.Revista Telos, URBE. Vol. 18 (1). Maracaibo, Venezuela. (Pp. 138-158). Disponible en: http://www.redalyc.org/pdf/993/99344833009.pdf Consulta 12/10/2017.

D’Angelo, Ovidio (2005). Autonomía integradora y transformación social: El desafío ético emancipatorio de la complejidad. Editorial CIPS, Cuba.

Diccionario Manual de la Lengua Española (2009). Editorial Larousse. España.

Elliot, John (2000). El cambio Educativo desde la Investigación Acción. (Segunda edición). Editorial Morata. España.

El Troudi, Haiman, Harnecker, Marta y Bonilla-Molina, Luís (2005). Herramientas para la participación. Ministerio de la participación Popular. Venezuela.

Freire, Paulo (1993). Pedagogía de la esperanza. Editorial Siglo XXI. México.

Freire, Paulo (2005). Pedagogía del Oprimido. Traducido por Jorge Mellado. (Quincuagésima quinta edición en español). Siglo XXI Editores: México.

García, José, Mena, Juan, y Sánchez, Emilio (2011). Investigación-reflexión-acción y asesoramiento: análisis de las reflexiones de dos orientadoras en su contexto de trabajo. Revista de Educación. N ${ }^{o}$ 356, Septiembre-Diciembre. España. (Pp. 253-278).

Hartzler, Meg y Henry, Jane. (1999). Teoría y aplicaciones del trabajo en equipo. Editorial Oxford. México.

Kemmis, Stephen. y McTaggart, Robin. (1988). Cómo planificar la Investigaciónacción. Editorial Alertes. España.

Latorre, Antonio (2007). La investigación-acción. Conocer y cambiar la práctica educativa. Editorial Graó. España.

López, Hilda. (2001). Cambiando a través de la Investigación Acción Participativa. EdiciónXdemanda. Venezuela.

Maldonado, María Ofelia. (2005). Escuelas básicas como comunidades de aprendizaje para la formación del ciudadano participativo. Revista Venezolana de Ciencias Sociales, Vol. 9 No 1, Venezuela. (Pp. 204-216).

Manzanilla, Orestes (2005). Gerencia de la participación ciudadana. (Una visión integralista). (Primera Edición). Editorial Tipografía Principios. Venezuela.

Martí, Joel, Pascual, Jordi, y Rebollo, Oscar (Coord.) (2005). Participación y desarrollo comunitario en medio urbano. Experiencias y reflexiones. Editorial CIMAS. España. 
Matos, Yuraima, Pasek, Eva y Rojas, Yurmely. (2008). La Investigación como trabajo en equipo desde las experiencias y reflexiones de los actores. Revista Copérnico. Año IV, No. 8. Enero-junio, 2008. Venezuela. (Pp. 03-10).

Matos, Yuraima, Pasek, Eva y Rojas, Alexis (2014). Actividades para favorecer la participación de los padres y representantes en el preescolar. Revista Sustentabilidad al Día. Año 2014, Nº 1. UVM, Valera, Trujillo, Venezuela. (Pp. 1-21).

Montero, Maritza (2006). Hacer para transformar: El método en la psicología comunitaria. Tramas sociales, 35. Editorial Paidós. España.

Mora, Héctor (1991). Autogestión y Capacitación en el Perú: Las cooperativas agrarias de Producción en el Valle Sagrado de los Incas. Editorial EUNED. Perú.

Pérez, Gloria (1994). Investigación Cualitativa. Retos e interrogantes. IMétodo. Editorial La Muralla. España.

Pérez, Gloria (2002). Elaboración de proyectos sociales. Casos prácticos. Editorial Narcea. España.

Pérez, Ángel, Barquín, Javier y Angulo, Félix. (1999). Desarrollo profesional del docente: Política, investigación y práctica. Ediciones Akal. España.

Quiroga, Hiram, Quevedo, Santiago, y Chiriboga, Eduardo (2003). Hacia el cambio mediante la autogestión comunitaria. CONADE. Ecuador.

Revueltas, José (2008). México 68: Juventud y Revolución. Editorial Era. México.

Rodríguez, Lidia (2003). Producción y transmisión del conocimiento en Freire. Editorial CLACSO. Argentina.

Sánchez, Manuel. (2001). La participación. Metodología y práctica. EditorialPopular. España.

Scribano, Adian (2007). El proceso de investigación social cualitativo. Editorial Prometeo. Argentina.

Shermerhom, John (2008). Comportamiento Organizacional. Editorial Limusa, México.

Véliz, Arnoldo (2008). Proyectos comunitarios e Investigación cualitativa. (Primera Edición). Editorial Texto. Venezuela. 